\title{
ARTICLE
}

\section{Differences in Calibration Factors of a Neutron Diode Dosimeter at Different Facilities}

\author{
Namho Lee ${ }^{1 *}$, Seungchan $\mathrm{Oh}^{1}$, Hyunjin Lee ${ }^{1}$, Younggwan Hwang ${ }^{1}$, Geunuck Youk ${ }^{2}$ \\ ${ }^{1}$ Korea Atomic Energy Research Institute, Duckjin-Dong, Yousung-Ku, Taejon, Korea \\ ${ }^{2}$ ServeBot Co., Fairfax, VA 22030, USA
}

\begin{abstract}
The calibration of neutron diodes as fast neutron dosimeters was undertaken at four different facilities. The results show significant variations in calibration factors, ranging from 1.4 to $14.7 \mathrm{mV} / \mathrm{rad}$, across the four facilities. This is partially attributable to dose-rate dependency due to annealing of radiation induced defects. Further investigations on other potential reasons for the discrepancy is recommended, as well as call for additional international efforts in providing more standardized dosimetry services using accelerator-based high-energy neutron generators.
\end{abstract}

\section{KEYWORDS: neutron diode, dosimeter calibration, fast neutron dosimetry, dose-rate, $14 \mathrm{MeV}$, Cf-252 source}

\section{Introduction}

Specially developed PIN diode sensors for a personal dosimeter in a fast neutron environment have extensively been tested at credible facilities worldwide. There was an order of magnitude difference in dose-rates among facilities. The differences in the resultant sensor response values among facilities are not ignorable. This paper will discuss dose-rate effect among other potential reasons for the differences.

In the era of personal electronic gadgets, people prefer an electronic dosimeter that can display instant reading, versus a passive dosimeter (such as film badges and TLD badges) which are usually only read once in several weeks. This is especially true for neutron dosimetry for which miniaturized sensor elements are still emerging. Among widely spread energy ranges, fast neutrons are hard to detect due to their low interaction cross sections. Many centimeters thick of moderator, to reduce their energy, is impractical as a wearable personal dosimeter. Fortunately, silicon-diode-neutron dosimeters made it possible. More sensitive neutron diodes than commercially available ones were manufactured after parametric studies about diode geometry and about semiconductor fabrication processes.[1]

\section{Detection Principle}

When a fast neutron collides with a silicon atom of a silicon lattice of a PIN diode, its energy is transferred to the constituent elements of the lattice and lattice defects are produced by scattered recoil nuclei. The recoil nuclei with enough kinetic energy produce again another group of lattice defects. The defects are in the form of vacancy and interstitial. The lattice defects become trap centers for electric charges, and consequently change the I-V(Current-Voltage) characteristic curve of the PIN diode.[2]

The defect centers inside a silicon PIN diode act as recombination centers of charges moving along electric fields. They cause the decrease of the lifetime of minority carriers injected to depletion region, thus decreasing the diode current.

The number of defect centers increase with the dose of

*Corresponding Author, E-mail:nhlee@kaeri.re.kr

(C) Atomic Energy Society of Japan neutron irradiation. The relation between the increase of neutron dose and the reduction of minority carrier lifetime is as follows:

$$
1 / \tau=1 / \tau_{0}+k \varnothing
$$

Here, $\tau_{0}$ is initial carrier lifetime, $\tau$ is carrier lifetime after neutron irradiation, $\varnothing$ is neutron fluence, and $k$ is damage constant at base region. Because the density distribution of carriers injected into the depletion region is the function of base width and diffusion length, $\mathrm{L}=(\mathrm{D} \tau)^{1 / 2}$, L: ambipolar diffusion length, D: diffusion constant), neutron irradiation reduces the carrier lifetime and the length of diffusion.

In addition, because the external voltage applied to a PIN diode is expressed as a proportional relationship like Equation (2)[3],[4], neutron fluence in the above Equation (1) is inversely proportional to the charge of carrier lifetime in the device. The applied voltage is inversely proportional to the carrier lifetime from the relation of diffusion length and Equation (2). Consequently, the $V_{D}$ is proportional to neutron fluence.

$$
V_{D} \propto\left(\frac{W}{L}\right)^{2}
$$

Here, $\mathrm{W}$ is the diode base width, and $\mathrm{V}_{\mathrm{D}}$ is the biased voltage. Such a relation, in which the forward voltage of a diode increases with neutron fluence, is expressed by the rightward shift of the I-V characteristic curve of the device. The amount of electric changes in the characteristic curve shifted by neutron irradiation can be measured by observing the increase of voltage at a constant current or the decrease of current at a constant voltage. It is then possible to find the dose of cumulative neutron irradiation simply by measuring the voltage for a constant current or the current for a constant voltage.

\section{Development of PIN Diode Dosimeter}

In the development of a radiation dosimeter, sensitivity improvement is crucial because it improves measurement accuracy. In particular, it is known that the geometric 
structure of a diode is closely related to neutron sensitivity. [5] To identify geometrical factors affecting neutron sensitivity, we manufactured test diodes with different geometry followed by performance tests in a neutron field. The result shows that the characteristic of PIN diodes changes linearly by the neutron accumulative dose. Based on the test results, effect of diode geometry on neutron sensitivity was analyzed. It shows that neutron sensitivity gets higher when layer I gets thicker. It also shows that neutron sensitivity is in inverse proportion with the cross sectional area of a device. It can be concluded that a PIN diode that has thick I layer and narrow cross section is more sensitive to neutrons.

To supplement the neutron tests, numerical simulations were performed to examine neutron sensitivity change due to diode's geometrical variations. Swartz et al.[6] described the changes in external current-voltage characteristics caused by displacement damage when PIN diodes are irradiated with fast neutrons. Using his expression, diode's neutron sensitivity to a constant neutron dose was calculated by varying " $d$ " the thickness of PIN diode and " $A$ " the cross section of the device. The results of the present simulation show that a structure with large thickness $(d)$ and narrow cross section $(A)$ is necessary to make PIN diodes sensitive to neutrons. These results are similar in trend to that of the experiment described in the previous section. However, if a PIN diode is too thick, it causes uneven characteristics of the device as well as excessive power consumption owing to the increase of voltage. If the cross section of a device is too narrow, it increases surface current leakage, which in turn lowers neutron sensitivity. Thus it is important to find optimal values of those variables.

Based on the results of simulations and of experiments on the various device geometry as presented above, we manufactured discrete PIN diodes (Fig. 1) using a $1.2 \mathrm{~mm}$ thick high-purity wafer, of which resistance was 3,000 $\Omega . \mathrm{cm}$ and the length of a side of the cross section was $1 \mathrm{~mm}$.

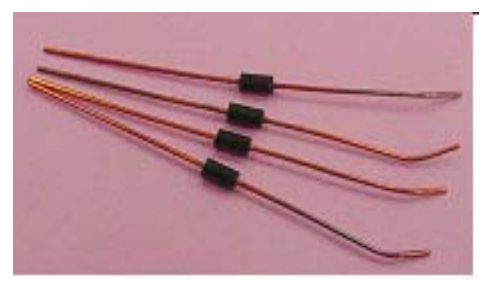

Fig. 1 Manufactured PIN diodes

\section{Dosimeter Calibration}

\section{Using 14.8 MeV D-T neutron generator in Europe}

The irradiations were performed in open geometry in the low-scatter measurement hall $(24 \mathrm{~m} \times 30 \mathrm{~m} \times 14 \mathrm{~m})$ of a European accelerator facility. The $14.85 \mathrm{MeV}$ field was produced according to ISO standards.[7-9] The related parameters used are listed in Table 1.1. The measurement quantities of the instrument under test are the ambient dose equivalent $H^{*}(10)$.
Table 1. Informational data on the monoenergetic neutron fields. The mean energy $\boldsymbol{E}_{\mathrm{n}}$ and the width $\boldsymbol{\Delta}_{\mathbf{n}}$ (FWHM) of the unscattered neutron distribution are nominal values. Standard measurement uncertainties $(k=1)$ are given for the ratio $\boldsymbol{\Phi}_{\text {sc }} / \boldsymbol{\Phi}_{\text {dir }}$ and for the fluence to ambient dose equivalent conversion factors for unscattered neutrons $\boldsymbol{h}_{\boldsymbol{\phi}}^{\boldsymbol{*} \text {,dir }}$ and scattered neutrons $\boldsymbol{h}^{*}{ }_{\boldsymbol{\Phi} \text {,sc }}$ The uncertainties of the conversions factors account for the uncertainty introduced the folding with the spectral neutron fluence $\boldsymbol{\Phi}_{E}$.

\begin{tabular}{|c|c|c|c|c|c|}
\hline Reaction & $\begin{array}{c}E_{\mathrm{n}} \\
\mathrm{MeV}\end{array}$ & $\begin{array}{c}\Delta E_{\mathrm{n}} \\
\mathrm{MeV}\end{array}$ & $\left(\Phi_{\mathrm{sc}} / \Phi_{\mathrm{dir}}\right)$ & $\begin{array}{c}h^{*} \Phi, \operatorname{dir}(10) \\
\text { pSv } \mathbf{c m}^{2}\end{array}$ & $\begin{array}{c}h^{*} \Phi, \mathrm{sc}(10) \\
\mathrm{pSv} \mathrm{cm}^{2}\end{array}$ \\
\hline${ }^{3} \mathrm{H}(\mathrm{d}, \mathrm{n}){ }^{4} \mathrm{He}$ & 14.85 & 0.45 & $0.016(4)$ & $5.36(4) \cdot 10^{2}$ & $0.45(11) \cdot 10^{2}$ \\
\hline
\end{tabular}

The total neutron fluence $\Phi$ is the sum of the fluence $\Phi_{\text {dir }}$ of the direct neutrons and of the fluence $\Phi_{\text {sc }}$ of neutrons scattered in the target assembly. The fluence of unscattered neutrons $\Phi_{\text {dir }}$ at the reference position was measured using a recoil proton telescope. Details of the measurement and analysis procedures are described in $[10,12]$. The fluence of neutrons scattered in the target was calculated using the Monte Carlo code TARGET [6].

The $\mathrm{H}^{*}(10)$ is the sum of the $\mathrm{H}^{*}{ }_{\text {dir }}(10)$ of the unscattered neutrons and the $\mathrm{H}^{*}{ }_{\mathrm{sc}}(10)$ of the neutrons scattered in the target assembly. $\mathrm{H}^{*}{ }_{\text {dir }}(10)$ and $\mathrm{H}^{*}{ }_{\mathrm{sc}}(10)$ are calculated from $\Phi_{\text {dir }}$ and $\Phi_{\text {sc }}$ using the conversion factors $h^{*}{ }_{\Phi, \text {,ir }}(10)$ and $\mathrm{h}_{\Phi, \mathrm{sc}}(10)$. The values for $\mathrm{h}^{*}{ }_{\Phi_{\text {,dir }}}(10)$, taken from [6], are identical to ICRP-publication no. 74. The values for $\mathrm{h}^{*}{ }_{\Phi, \mathrm{sc}}(10)$ were calculated by folding the spectral neutron fluence $\Phi_{\mathrm{E} \text {,sc }}$ of scattered neutrons with the energy dependent conversion coefficients taken from ICRP-publication no. 74.. With exception of Table 1.1, the uncertainties in this report are expanded uncertainties according to DIN 1319, part 3 with a coverage factor $\mathrm{k}=2$, providing a level of confidence of approximately $95 \%$. They were determined according to the "Guide to the Expression of Uncertainty in Measurement" (ISO 1995).

Irradiation was also performed. Average dose-rate was $5.3 \mathrm{cGy} / \mathrm{h}$. Resultant ADC value has increased at most 4 5 that is about $62.4 \sim 78 \mathrm{mV}$ worth. Therefore, dosimeter sensitivity to $14.5 \mathrm{MeV}$ neutrons becomes $62.4 \mathrm{mV} / 50 \mathrm{cGy}=1.28 \mathrm{mV} / \mathrm{cGy} \sim$ $1.56 \mathrm{mV} / \mathrm{cGy}$. This sensitivity value and dose-rate are, so far, the lowest among results from the other three irradiation facilities.

At this low dose-rate condition, defect generation rate is so low that there is enough time to recover. A significant portion of neutron-induced defects can be annealed during irradiation. If sufficient annealing was taken place during irradiation, there may not be sufficient room to anneal, even after one set of irradiation ends. Thus, diode conductivity will not change even after an over-night pause of irradiation. To demonstrate that this is the case for the low sensitivity, a $2^{\text {nd }}$ batch of irradiation data are put together with the $1^{\text {st }}$ batch of irradiation. The result does not show any sign of change during and following an overnight pause. No sudden drop of $\mathrm{ADC}$ value due to annealing can be observed. This demonstrates the annealing during irradiation that has lowered dosimeter sensitivity.

\section{Using $14.8 \mathrm{MeV}$ D-T neutron generator in USA}

A $1.18 \mathrm{~g}$ copper foil with 1 " diameter was exposed to mono-energetic $14.8 \mathrm{MeV}$ neutrons at about 2" from the source 
for 184 seconds. The neutron generator was operated to maintain $2 \mathrm{~mA}$ at $125 \mathrm{kV}$. Cu foil activation method was used to measure neutron fluence at the diode location. The number of radiation counts under the $0.51 \mathrm{MeV}$ peak, which was accumulated for 60 sec, was 240,638 counts. The measured detection system efficiency was $27.4 \%$. After the efficiency correction, the calculated neutron flux for 184 second irradiation interval was $4.9 \times 10^{7} \mathrm{n} / \mathrm{cm}^{\wedge} 2$-sec which is equivalent to $1261.3 \mathrm{cGy} / \mathrm{h}$ using the ICRP-74 conversion factor $1 \mathrm{n} / \mathrm{cm}^{\wedge} 2=7.15 \times 10^{-9} \mathrm{rad}$ (tissue). With the rads to rem conversion factor of 7.5 for $14 \mathrm{MeV}$ neutrons, the diode performance was calculated as 7.41 $\mathrm{mV} / \mathrm{rad}$ (Tissue).

\section{Using neutrons with average energy of $10.2 \mathrm{MeV}$}

The PIN diodes were irradiated by the secondary neutron that occurs when an accelerated proton with $35 \mathrm{MeV}$ is reacted with a Beryllium $(\mathrm{Be})$ target in the cyclotron (MC-50) in Korea. The neutron energy has the form of Gaussian statistic distribution with maximum $35 \mathrm{MeV}$ and average value of $10.2 \mathrm{MeV}$. The seven PIN diodes developed in this study were irradiated at the same time for comparison. The PIN diode performance was very linear with neutron dose, as shown in Figure 3.3. As shown in the Figure, the average sensitivity was $17.3 \mathrm{mV}$ per unit neutron dose (1 cGy). The discrepancies between diodes were less than $8 \%$.

Later, calibration of the irradiator was performed with multiple Bonar sphere detectors and Monte-Carlo analysis. This revealed that the revised dose of the facility was just a quarter of the value used for the Fig. 2. Therefore, the resultant sensitivity becomes $17.3 \mathrm{mV} / \mathrm{cGy} \quad \mathrm{x} \quad 0.25=4.3$ $\mathrm{mV} / \mathrm{cGy}$.

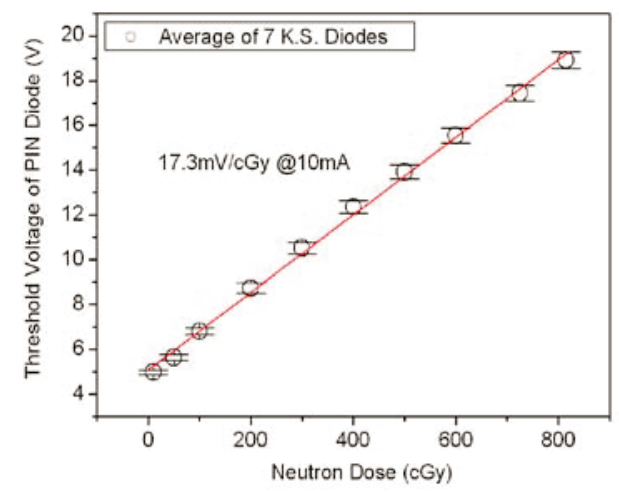

Fig. 2 Dosimeter diode sensor performance in a continuous spectrum neutron environment with average $10.2 \mathrm{MeV}$.

\section{Using a Cf-252 Fission Neutron Source}

To know dosimeter sensor behavior at a nuclear fission environment, the neutron diodes were irradiated at a Cf-252 national standard source at a US national institute. At the facility, all the major structural materials that can reflect neutrons are at least $2 \mathrm{~m}$ away from the Cf-252 source. Moreover, to reduce contamination from scattered neutrons with low energy, specially constructed walls -- filled with neutron observing boron -- reduce additional low-energy neutrons.

Fig. 3 shows radiation performance of diode sensors in a
Cf-252 fission neutron environment. Results show excellent linearity with neutron dose. The diode sensitivity to neutron dose was $14.63+0.13 \mathrm{mV} / \mathrm{rad}$ based on fission neutron dose given by the institute.

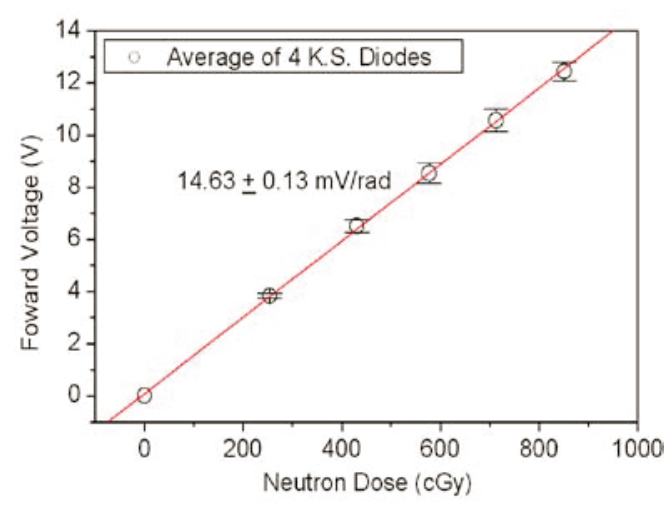

Fig. 3 Dosimeter diode sensor performance in a Cf-252 fission neutron environment. Results show excellent linearity with neutron dose.

\section{Summary of Results}

Dosimeter sensitivity (calibration factor) of a PIN diode that were specially designed and fabricated for fast neutron dosimetry was measured at four different facilities using four different sources. In the sensitivity measurements, constant current pulses of $10 \mathrm{~mA}, 10 \mathrm{msec}$ pulse widths were used to minimize unwanted heating by the measurement current. The final values of dosimeter sensitivity to high-energy neutrons at the worldwide facilities are compared with each other in Table 5-1. Showing also are related test conditions and bases of calculations.

Table 1. Irradiation performance and related conditions for the tests at various facilities. The energy of fission neutrons was not enough to produce considerable spallation reactions unlike those neutrons at other facilities.

\begin{tabular}{|c|l|l|l|l|}
\hline Lab. & Europe & USA & Korea & Cf-252 \\
\hline $\begin{array}{c}\text { Energy } \\
\text { (MeV) }\end{array}$ & 14.8 & 14.8 & $\begin{array}{l}35>\text { Avg. } \\
10.6\end{array}$ & $\begin{array}{l}2.27>\text { Avg. } \\
1.17\end{array}$ \\
\hline $\begin{array}{c}\text { Intensity } \\
\text { (n/cm } \mathbf{s}^{2} \text { ) }\end{array}$ & $1.7 \times 10^{5}$ & $4.9 \times 10^{7}$ & $\begin{array}{l}* 1.6 \times 10^{7} \\
\text { (Calculated) }\end{array}$ & $1.0 \times 10^{7}$ \\
\hline $\begin{array}{c}\text { Conversion } \\
\text { Factor } \\
\text { (rad(tissue)/n/c } \\
\mathbf{m}^{2} \text { ) \& } \\
\text { Reference }\end{array}$ & $\begin{array}{l}7.15 \times 10^{-9} \\
\text { ICRP-74 }\end{array}$ & $\begin{array}{l}7.15 \times 10^{-9} \\
\text { ICRP-74 }\end{array}$ & $\begin{array}{l}7.15 \times 10^{-9} \\
\text { (Assumed) } \\
\text { ICRP-74 }\end{array}$ & $\begin{array}{l}3.1 \times 10^{-9} \\
\text { ISO } 8529\end{array}$ \\
\hline $\begin{array}{c}\text { Quality Factor } \\
\text { S-T Distance } \\
\text { (cm) }\end{array}$ & 25 & 7.5 & 150 & 4.34 \\
\hline $\begin{array}{c}\text { Dose Rate } \\
\text { (cGy/h) }\end{array}$ & 5.3 & 1261.3 & 400 & 115 \\
\hline $\begin{array}{c}\text { Measurement } \\
\text { Current (mA) }\end{array}$ & 1.4 & 7.41 & 4.3 & $14.63 \pm 0.13$ \\
\hline $\begin{array}{c}\text { Sensitivity } \\
\text { (mV/cGy) }\end{array}$ & 1.4 & & $>20$ & $>11$ \\
\hline Error (\%) & - & & \\
\hline
\end{tabular}

The results show significant variations in diode sensitivities, ranging from 1.4 to $14.7 \mathrm{mV} / \mathrm{rad}$ at three different countries across the four facilities. Two $14 \mathrm{MeV}$ D-T generators in two different countries were used as neutron 
sources along with an accelerator with a maximum of $35 \mathrm{MeV}$ of neutron energy (average $18 \mathrm{MeV}$ ) and a Cf-252 source. Although the effects of different mean energies among the facilities are not negligible, differences in one order of magnitude were observed.

The first three high energy data are replotted in Figure 5.1. As shown in the Figure 5-1, sensitivity increases as dose-rate increases. The trend can be explained by the nature of time sensitivity of annealing mechanism of radiation damage. At low dose-rate, during irradiation, there is an enough time in which a part of radiation-induced damage can be annealed and recovered, resulting lowering its sensitivity. As the dose-rate becomes higher, less damage will be annealed, resulting higher sensitivity. It may not necessarily be a linear function. It can saturate at higher dose-rate due to inadequate annealing of radiation induced defect, simply because there is not enough time to anneal at a high dose rate.

As shown in the Fig. 4, sensitivity increases as dose-rate increases. The trend can be explained by the nature of time sensitivity of annealing mechanism of radiation damage. At low dose-rate, during irradiation, there is enough time in which a part of radiation-induced damage can be annealed and recovered, resulting in lowered sensitivity. As dose-rate becomes higher, less damage will be annealed, resulting in higher sensitivity. It may not necessarily be a linear function. It can saturate at a higher dose-rate due to inadequate annealing of radiation-induced defect, simply due to a lack of time to anneal at high dose rate.

The results are a compilation of different dose-rates at different facilities. The point of dose-rate dependency can be made stronger if we can vary dose-rate only at a facility. Further experimental study on dose-rate effect, while maintaining all other variables constant except the dose-rate, is recommended as well as supporting analytical calculations.

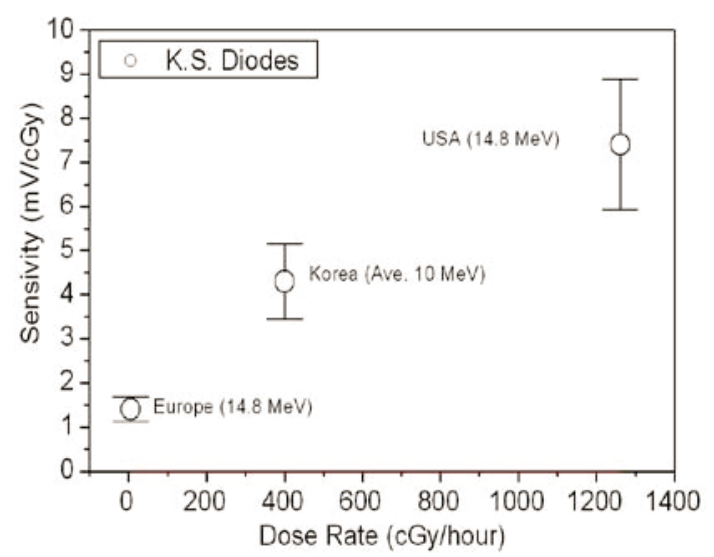

Fig. 4 Dosimeter sensitivity to high energy ( $>10 \mathrm{MeV}$ ) neutrons showing potential dose-rate effect. Diode sensitivity to neutrons increased as neutron-beam intensity increased.

\section{Conclusion}

Dosimeter sensitivity of a PIN diode was measured at four different facilities using four different sources. The results show significant variations in diode sensitivities, ranging from 1.4 to $14.7 \mathrm{mV} / \mathrm{rad}$ at three different countries across the four facilities. Higher the intensity of neutrons at a facility was, more sensitive the sensors were to neutrons. Considering room-temperature annealing of radiation-induced damages, a certain correlation between the dosimeter response and dose-rate is expected. A diode performance vs. dose-rate graph shows a proportional relationship between the two. A lesson learned was that dose-rate should carefully be considered as an important test condition when annealing sensitive sensors are tested. It is especially true when a dose calibration of a facility is compared with another facility using a annealing sensitive dosimeter. Further investigation on this matter is recommended. Even if variations among calibration methods and dose-rate dependency are considered as contributing factors, the magnitude of the differences observed is alarming. An important focal point of the paper is the raising of a call for additional international efforts in providing more standardized dosimetry services using accelerator based high-energy neutron generators.

\section{References}

1) S. H. Kim, N. H. Lee, "Development of Semiconductor Neutron Dosimeter with a PIN Diode", J. Nucl. Sci, and Tech., (2004).

2) George C. Messenger et al, The Effect of Radiation on Electronic System, Van Nostrand Reinhold Company Inc., (1986).

3) M. Valdinoci, et al. "Analysis of Conductivity Degradation in Gold/Platinum-Doped Silicon," Trans. On Electron Devices, 43[12], 2269 (1996).

4) M. McPherson, T. Sloan, B. K. Jones, "Suppression of Irradiation Effect in Gold-Doped Silicon Detectors", J. Phys. D: Appl. Phys. 30, 3028 (1997).

5) O. J. Mengali, E. Paskell, R. W. Beck, and C. S. Peet, "The Use of Diffused Junction in Silicon as Fast-Neutron Dosimeters," Proc. of 2nd Conf. on Nuclear Radiation Effects on Semiconductor Devices, Materials and Circuits (1959).

6) John M. Swartz and Marlin O. Thurston, "Analysis of the Effect of Fast- Neutron Bombardment on the Current-Voltage Characteristic of a Conductivity-Modulated P-I-N Diode," J. Appl. Phys. 37[2], 745 (1966).

7) International Standard ISO 8529-1, Reference neutron radiations: Characteristics and methods of production (2001).

8) International Standard ISO 8529-2, Calibration fundamentals of radiation protection devices related to the basic quantities characterizing the radiation field (2002).

9) International Standard ISO 8529-3, Reference neutron radiations: Calibration of area and personal dosimeters and determination of their response as a function of neutron energy and angle of incidence (1998).

10) D. Schlegel, S. Guldbakke, Neutron fluence measurements with a recoil proportional counter, PTB laboratory report PTB-6.41-2002-03, Braunschweig (2002).

11) D. Schlegel, S. Guldbakke, Neutron fluence measurements with a recoil proton telescope, PTB laboratory report PTB-6.41-2002-04, Braunschweig (2002).

12) D. Schlegel, TARGET user's manual, PTB laboratory report PTB-6.41-98-1, Braunschweig (1998). 